\title{
FIGO Committee for the Ethical Aspects of Human Reproduction and Women's Health
}

\section{Introduction}

The FIGO Committee for the Ethical Aspects of Human Reproduction and Women's Health considers the ethical aspects of issues that impact the discipline of Obstetrics, Gynecology and Women's Health. The following documents represent the result of that carefully researched and considered discussion. This material is not intended to reflect an official position of FIGO, but to provide material for consideration and debate about these ethical aspects of our discipline for member organizations and their constituent membership.

\section{Definition of Pregnancy}

Natural human reproduction is a process which involves the production of male and female gametes and their union at fertilization. Pregnancy is that part of the process that commences with the implantation of the conceptus in a woman ${ }^{1}$, and ends with either the birth ${ }^{2}$ of an infant or an abortion ${ }^{3}$.

Cairo, March 1998

\section{Ethical Guidelines Regarding Interventions for Fetal Well-Being}

1 Most women will act to improve their chance of having a normal birth and healthy baby if they have access to the necessary information and support.

2 Extending care to the fetus by giving the pregnant woman the support she needs provides the best hope of enhancing the well-being of both the fetus and the mother-to-be.

Verification of this is usually only possible at the present time at 3 weeks or more after implantation.

2 WHO definition of a birth: 22 weeks' menstrual age or more.

In some cases the dead products of conception may be reabsorbed or retained.

\begin{tabular}{ll}
\hline KARGER & $\odot 1999$ S. Karger AG, Basel \\
Fax +41 61 3061234 & 0378-7346/99/0482-0073\$17.50/0 \\
E-Mail karger@karger.ch & Accessible online at: \\
www.karger.com & http://BioMedNet.com/karger
\end{tabular}

FIGO Committee for the Ethical Aspects of Human Reproduction and Women's Health Secretariat: 27 Sussex Place, Regents Park,

London NW1 4RG (UK

Tel. +44 171723 2951, Fax +44 171258 0737, E-Mail secret@figo.win-uk.net 
3 Although the fetus may benefit from care, it is completely dependent on the mother and any treatment must be through her body.

4 While the majority of women act in a way that provides a healthy environment and are even ready to take risks on behalf of their fetus, there may be situations where their interests do not coincide:

a The mother's behaviour may create risks for herself and her fetus (e.g. use of drugs, tobacco, and alcohol, not attending appropriately provided antenatal care, etc.).

b The mother may choose not to accept diagnostic, medical or surgical procedures aimed at preserving fetal well-being, including caesarean section for fetal indications.

5 The medical team has a responsibility to fully inform the mother, to counsel her with empathy and patience, and to provide such support services as are needed. She may then accept the recommended medical advice and/or change her life-style.

6 However, no woman should be forced to undergo an unwished-for medical or surgical procedure in order to preserve the life or health of her fetus, as this would be a violation of her autonomy and fundamental human rights.

7 Resort to the courts or to judicial intervention when a woman has made an informed refusal of medical or surgical treatment is inappropriate and usually counter-productive.

8 If maternal competence for medical decision-making is impaired, health care providers should act in the best interests of the woman first and her fetus second. Information from the family and others may help to ascertain what she would have wished.

9 The wishes of pregnant minors who are competent to give informed consent regarding medical and surgical procedures should be respected. When they are not considered competent, the advice of the next of kin and, if appropriate the courts, should be considered before determining management.

Cairo, March 1998

\section{Ethical Aspects Regarding Caesarean Delivery for Non-Medical Reasons}

1 The medical profession throughout the world has been concerned for many years at the increasing rate of caesarean delivery. Many factors, medical, legal, psychological, social and financial have contributed to this increase. Efforts to reduce the excessive use of this procedure have been disappointing.

2 Caesarean section is a surgical intervention with potential hazards for both mother and child. It also uses more health care resources than normal vaginal delivery.

3 Physicians have a professional duty to do nothing that may harm their patients. They also have an ethical duty to society to allocate health care resources wisely to procedures and treatments for which there is clear evidence of a net benefit to health. Physicians are not obligated to perform an intervention for which there is no medical advantage.

4 Recently in some societies obstetricians have had increasing requests from women to be delivered by caesarean section for personal rather than for medical reasons.

5 At present there is no hard evidence on the relative risks and benefits of term caesarean delivery for nonmedical reasons, as compared with vaginal delivery. However, available evidence suggests that normal vaginal delivery is safer in the short and long term for both mother and child. Surgery on the uterus also has implications for later pregnancies and deliveries. In addition there is also a natural concern at introducing an artificial method of delivery in place of the natural process without medical justification.

6 Physicians have the responsibility to inform and counsel women in this matter. At present, because hard evidence of net benefit does not exist, performing caesarean section for non-medical reasons is ethically not justified.

London, September 1998

\section{Ethical Guidelines Regarding the Management of Pregnancy Related to Sudden Unexpected Maternal Death}

1 Once a pregnant woman has been declared dead because of brain death, or death is imminent due to lack of circulatory and respiratory functions, the life and well-being of her fetus become a matter of urgent consideration.

2 Pending a decision on management, every effort should be made to maintain the woman's circulation and ventilation unless it is known to be against her recent and clearly expressed wish. 
3 The possible options are:

a Immediate caesarean section.

b Continuation of efforts to maintain the circulatory and respiratory functions of the woman to allow her fetus to mature.

c Discontinuation of support for the woman's circulation and ventilation.

4 Among the issues to be considered are:

a The viability of the fetus.

b The probable health status of the fetus.

c Any wish expressed by the mother.

d The views of her partner and/or family members.

5 In case of inability to sustain support of the woman's vital organ functions, there should be immediate recourse to caesarean delivery provided that none of the exceptions below pertain, since delay increases the chance of damage to the fetus:

a Such an action would be against the woman's recent and clearly expressed wish.

b The fetus is too immature, or has probable poor health status.

6 Maintaining the pregnancy in order for the fetus to become more mature requires counselling to enable informed consent of the woman's partner and/or family members.

London, September 1998

\section{Ethical Guidelines Regarding the Procedure of Collecting Cord-Blood for Banking}

1 The discovery that umbilical cord-blood provided a rich source of haemopoietic stems cells used in transplantation in diseases such as leukaemia, has led to the organised collection of blood from this source and its retention in cord-blood banks until required.

2 In some countries the process of collecting, banking and using the cord-blood of term infants has been commercialised.

3 It is ethically necessary for the mother to give informed consent (before delivery) for the collection of cordblood or banking.

4 The information mothers currently receive at the time of requesting consent is that blood in the placenta is no longer of use to the baby and this 'waste blood' may help to save another person's life. This information is incomplete and does not permit informed consent.

5 Early clamping of the umbilical cord following vaginal delivery is likely to deprive the newborn infant of at least a third of its normal circulating blood volume, and it will also cause a haemodynamic disturbance. These factors may result in serious morbidity.

6 For consent to be informed, the harmful effects of early cord clamping should be disclosed and the mother assured that the collection of cord-blood will not involve early clamping.

7 In summary, permission to collect blood from the cord for banking should not lead to clamping of the cord earlier than 20-30 s after delivery of the baby.

Cairo, March 1998

\section{Ethical Guidelines Regarding Cloning}

1 The cloning of a sheep by nuclear transfer published in 1997 demonstrated that asexual reproduction of mammals can be brought about with the possibility that it may also work in humans. Cloning of mammals by another method, embryo splitting, has been possible for some time.

2 It is of note that there are at least three areas of the use of cloning in which the ethical and social issues that are raised differ, and should therefore be responded to differently. These are:

a Nuclear transfer cloning or embryo splitting in animals.

b Nuclear transfer cloning to produce human cell lines or tissues.

c Nuclear transfer cloning or embryo splitting to produce human beings (reproductive cloning).

3 Some human characteristics are strongly determined by DNA (e.g. blood group or HLA haplotype) whereas others, because they are a result of a complex interplay between genetic endowment and the rearing social and physical environment, are not (e.g. intelligence). Therefore, a human person coming from cloning would be identical to their source in some respects but not others.

4 With regard to reproductive cloning in humans, producing individuals by nuclear transfer cloning because they are genetic copies of identified adults does not respect human identity and individuality. It objectifies human beings, and as well as having unknown physical risks is likely to be psychologically harmful to individuals produced this way. The technique allows people with some predetermined characteristics to be made. This aspect means the technology can be used in an exploitative way (e.g. to produce a matched organ donor). 
5 For those wishing to form families there are other options available (for example, donor insemination, some new reproductive technologies, and adoption). Permitting infertile couples to use reproductive cloning (by either cloning method) to produce a child, breaches a natural barrier, which once passed, leaves no clear place to stop.

6 Although most people do not wish to see cloning permitted, there will be pressure favouring human reproductive cloning arising from specific personal and financial interests. In setting policy, these pressures should not be allowed to override the larger community's interests.

7 Given the nature of societies, the lack of regulatory structures in most countries, and the fact that some individuals would benefit from instrumentalizing replicands, cloning for the purpose of implantation into the uterus for development of a pregnancy should be prohibited.

8 Non-reproductive cloning research using somatic nuclei transferred into human oocytes should only be permitted in a regulated and accountable manner in licensed facilities. Research to produce particular cell lines would be acceptable provided that development beyond 14 days does not occur ${ }^{1}$.

9 A woman who has a mitochondrial defect, of necessity is at risk of passing this defect on to her offspring. Supplementation of the protoplasm of her oocytes by transfer of cytoplasm containing mitochondria does not constitute reproductive cloning. However, if one of her somatic cell nuclei were used to insert into another woman's oocyte, this would constitute reproductive cloning and be unacceptable.

10 Legislation or regulation prohibiting cloning to produce human persons (human reproductive cloning) is needed. However, it should be worded carefully so as not to prohibit other uses of cloning techniques in research. Such research promises to bring greater understanding of genetic control of differentiation, will contribute important knowledge, and ultimately be of benefit in therapeutic applications.

Cairo, March 1998

\section{Ethical Guidelines Regarding Induced Abortion for Non-Medical Reasons}

1 Induced abortion may be defined as the termination of pregnancy using drugs or surgical intervention after implantation and before the conceptus has become independently viable. (WHO definition of a birth: 22 weeks' menstrual age or more. $)^{1}$

2 Abortion is very widely considered to be ethically justified when undertaken for medical reasons to protect the life and health of the mother in cases of molar or ectopic pregnancies and malignant disease. Most people would also consider it to be justified in cases of incest or rape, when the conceptus is severely malformed, or when the mother's life is threatened by other serious disease.

3 The use of abortion for other social reasons remains very controversial because of the ethical dilemmas it presents to both women and the medical team. Women frequently agonize over their difficult choice, making what they regard under the circumstances to be the least worse decision. Health care providers wrestle with the moral values of preserving life, of providing care to women and of avoiding unsafe abortions.

4 In those countries where it has been measured, it has been found that half of all pregnancies are unintended and that half of these pregnancies end in termination. These are matters of grave concern, in particular to the medical profession.

5 Abortions for non-medical reasons when properly performed, particularly during the first trimester when the vast majority take place, are in fact safer than term deliveries.

6 However, the World Health Organization has estimated that nearly half of the 50 million induced abortions performed around the world each year are unsafe because they are undertaken by unskilled persons and/ or in an unsuitable environment.

7 The mortality following unsafe abortion is estimated to be very many times greater than when the procedure is performed in a medical environment. At least 75,000 women die unnecessarily each year after unsafe abor-
1 Note: This is not relevant to the lethally malformed fetus, cf. Ethical Aspects of the Management of the Severely Malformed Fetus. Int J Gynecol Obstet 1996;53:300. It is also important to consider Ethical Aspects in the Management of Newborn Infants at the Threshold of Viability. Int J Gynecol Obstet 1997;59:165-168. 
tion and very many more suffer life-long ill-health and disability, including sterility. ${ }^{2}$

8 Unsafe abortion has been widely practiced since time immemorial. Today it occurs mainly in countries with restrictive legislation with respect to the termination of pregnancy for non-medical reasons. Countries with poorly developed health services and where women are denied the right to control their fertility also have higher rates of unsafe abortion.

9 When countries have introduced legislation to permit abortion for non-medical reasons, the overall mortality and morbidity from the procedure has fallen dramatically, without any significant increase in terminations.

10 In the past most pregnancy terminations were undertaken surgically, however recent pharmaceutical developments have made it possible to bring about safe medical abortion in early pregnancy.

11 In addition, the reproductive process can be interrupted before pregnancy begins by classical contraceptive methods or by the more recently popularised emergency contraception. The latter is not an abortifacient because it has its effect prior to the earliest time of implantation. Nevertheless, these procedures may not be acceptable to some people.

\section{Recommendations}

1 Governments and other concerned organizations should make every effort to improve women's rights, status, and health, and should try to prevent unintended pregnancies by education (including on sexual matters), by counselling, by making available reliable information and services on family planning, and by developing more effective contraceptive methods. Abortion should never be promoted as a method of family planning.

2 Women have the right to make a choice on whether or not to reproduce and should therefore have access to legal, safe, effective, acceptable and affordable methods of contraception.
3 Providing the process of properly informed consent has been carried out, a woman's right to autonomy, combined with the need to prevent unsafe abortion, justifies the provision of safe abortion.

4 Most people, including physicians, prefer to avoid termination of pregnancy and it is with regret that they may judge it to be the best course, given a woman's circumstances. Some doctors feel that abortion is not permissible whatever the circumstances. Respect for their autonomy means that no doctor (or other member of the medical team) should be expected to advise or perform an abortion against his or her personal conviction. Their careers should not be prejudiced as a result. Such a doctor, however, has an obligation to refer the woman to a colleague who is not in principle opposed to termination.

5 Neither society, nor members of the health care team responsible for counselling women, have the right to impose their religious or cultural convictions regarding abortion on those whose attitudes are different. Counselling should include objective information.

6 Very careful counselling is required for minors. When competent to give informed consent, their wishes should be respected. When they are not considered competent, the advice of the parents or guardians and when appropriate the courts, should be considered before determining management.

7 The termination of pregnancy for non-medical reasons is best provided by the health care service on a nonprofit-making basis. Post-abortion counselling on fertility control should always be provided.

8 In summary, the Committee recommended that after appropriate counselling, a woman had the right to have access to medically or surgically induced abortion, and that the health care service had an obligation to provide such services as safely as possible.

Cairo, March 1998
2 WHO (1998) Unsafe Abortion: Global and Regional Estimates of Incidence of and Mortality due to Unsafe Abortion with a Listing of Available Country Data, ed 3. WHO/RHT/MSM/97.16.

FIGO Committee for the Ethical Aspects of Human Reproduction and Women's Health
Gynecol Obstet Invest 1999;48:73-77 